AperTO - Archivio Istituzionale Open Access dell'Università di Torino

\title{
Histological discrimination of fresh and frozen/thawed fish meat: European hake (Merluccius merluccius) as a possible model for white meat fish species
}

\section{This is the author's manuscript}

Original Citation:

\section{Availability:}

This version is available http://hdl.handle.net/2318/1669805

since 2018-06-19T12:07:54Z

Published version:

DOI:10.1016/j.foodcont.2018.04.056

Terms of use:

Open Access

Anyone can freely access the full text of works made available as "Open Access". Works made available under a Creative Commons license can be used according to the terms and conditions of said license. Use of all other works requires consent of the right holder (author or publisher) if not exempted from copyright protection by the applicable law. 
Control

Manuscript Number: FOODCONT-D-18-00609R1

Title: Histological discrimination of fresh and frozen/thawed fish meat: European hake (Merluccius merluccius) as a possible model for white meat fish species

Article Type: Research Paper

Keywords: Seafood fraud, Freezing-thawing, histology, Merluccius merluccius, white meat fish species

Corresponding Author: Dr. Andrea Armani,

Corresponding Author's Institution: University of Pisa

First Author: Lara Tinacci

Order of Authors: Lara Tinacci ; Andrea Armani; Alessandra Guidi; Daniele Nucera; Deborah Shvartzman; Vincenzo Miragliotta; Alessandra Coli; Elisabetta Giannessi; Maria R Stornelli; Baldassare Fronte; Francesco Di Iacovo; Francesca Abramo

Abstract: The present study aimed at setting up a standard operating histological procedure to discriminate fresh from frozen-thawed fish products of the species Merluccius merluccius (European hake). A preliminary histological analysis of fresh M. merluccius muscle was performed to select the sampling site and highlight possible timedependent tissue alterations during shelf-life. To set a suitable operational grid for discriminating the freezing process, morphological and morphometrical parameters were assessed on 90 muscle tissue samples collected from 30 fresh, 30 experimentally frozen at $-20^{\circ} \mathrm{C}$ and 30 Individual Quick Frozen (IQF) specimens of M. merluccius. Structural score, presence of freezing vacuoles, a number of vacuoles per field higher than 1.12 and the presence of interstitial seroproteinaceous material, which had achieved statistical significance in group comparisons were chosen as freezing markers. Accuracy and repeatability, assessed on the analysis of two independent operators (on-training and expert), showed high analytical specificity and sensitivity and a concordant diagnostic performance regardless the operators expertise. The grid was finally validated by a single blind test on 30 additional M. merluccius commercial products and allowed the allocation of all the samples to fresh or frozen status without inconclusive results. The method could be profitably applied against fraudulent adulteration practices. 


\section{Dear Editor,}

please find enclosed the manuscript entitled "Morphological and morphometrical discrimination of fresh and frozen/thawed fish meat: European hake (Merluccius merluccius) as a possible model for white meat fish species" to be considered for publication in LWT - Food Science and Technology.

Freezing is the commonest technology applied to prolong fish preservation, although it may produce muscle physical-chemical modifications altering the product's quality and induce a higher spoilage rate of the frozen-thawed product. Thus, clear information is needed for guaranteeing consumer's safety and fair commercial practices. In this respect, the European legislator imposed the obligation to declare the process of freezing and thawing occurred before the sale by indicating the designation 'defrosted' on the product's label. Nevertheless, deliberate substitutions of fresh with frozen-thawed fish are still common fraudulent incidents.

The recognition of histological structural alterations represents a tool for discriminating freezing treatments. This approach, firstly proposed for the common carp meat has been recently applied to other species (gilthead, red mullet, swordfish, bonito, salmon, turbot, albacore, little tunny, rainbow trout and anchovy). Even though the method was confirmed as a highly sensitive and specific, the presence of unspecified microscopic alterations reduced the assay accuracy and precision leading to "non-conclusive results". In addition, possible microscopic alterations eventually occurring within the product's shelf-life were not assessed.

The present study aimed at providing a standard operating histological procedure to discriminate fresh and frozen-thawed fish. The procedure, set and validated on M. merluccius (European hake), a species never analyzed until today, was thought to be extended to the analysis of the white meat fish category. A preliminary analysis of the muscular tissue histology of 15 whole fresh specimens sampled at different shelf life time was conducted to highlight possible time dependent modifications and to select the tissue sampling site. Then, the operative procedure was set by the analysis of both morphological and morphometrical parameters on a total of 90 muscular tissue samples belonging to fresh and frozen exemplars for the selection of objective indices of freezing process. Four parameters (structural score, presence of vacuoles, presence of extracellular and intracellular seroproteinaceous material, number of vacuole per field) were included in the final operative grid after a statistical analysis. The histological grid accuracy and repeatability assessed on the analysis of two distinct operators confirmed high specificity and sensitivity of the method and a high diagnostic concordance irrespective of the previous operators' skill. The method, validated by a single-blind test on 30 additional commercial products, was confirmed as a reliable check tool to be applied against the occurrence of fraudulent incidents and for the monitoring of the 
quality of the freezing process both in seafood business operator self-check monitoring and official controls.

The manuscript has not been published elsewhere nor is it being considered for publication elsewhere. All authors contributed to the intellectual or technical content of the study and to the drafting of the article. Finally, each of the listed authors approved this manuscript, agree to the order in which their names are listed, declare that no conflict of interests exists and disclose any commercial affiliation.

Best regards

Andrea Armani 
Dear Editor,

We are sending back the revised version of the paper FOODCONT-S-1800800. The title has been changed according to the reviewer's request. New title: "Histological discrimination of fresh and frozen/thawed fish meat: European hake (Merluccius merluccius) as a possible model for white meat fish species"

Here you can find our answers to reviewer's comments.

Reviewers' comments:

Reviewer \#1: This work presents a characterization of freezing effect on muscle structure using histological technique on Hake and a methodology to discriminate fresh and freeze/thaw products in order to propose a method to avoid fraudulent sales.

The paper is well written and the study very well conducted from the sampling protocol to the statistical analysis. The results are well discussed with a relevant bibliography.

In conclusion only minor revisions would be needed before this manuscript could be considered for publication.

We really thank the reviewer for appreciating the paper.

Minor revision

The title can be a little bit confusing, especially the term morphology is more appropriate to overall morphology of the fish, that was not be studied here. As only histological analysis have been performed I propose : "Histological discrimination of fresh and frozen/thawed fish meat: European hake (Merluccius merluccius) as a possible model for white meat fish species".

The title has been changed as suggested

In the Material and methods explain how the IQF fish are processed (degree, speed of freezing)

A brief description about the processing parameters has been included accordingly (Material and methods section lines 163-164)

Line 152: $567.073 \mathrm{\mu m}^{2}$ please verify the calculation this area correspond to a square image of $24 \mu \mathrm{m}$ side that not correspond to a 20X magnification.

It was a typing error. We now amended the manuscript erasing the dot.

The term Proteinaceous and moreover Seroproteinaceous seems not to be the right term, generally speaking muscle fiber comprise myofibrillar and sarcoplasmic protein, the soluble sarcoplasmic proteins corresponds probably to the proteinaceous material observed bit not the seroproteinacesous that comes from the blood sera, so use either sarcoplasmic or proteinaceous but not seroproteinaceous.

The term seroproteinaceous has been amended throughout the manuscript. 
Line 426 "for the analysis of the" instead of "for the analysis of to the"

Done 
Histological discrimination of fresh and frozen/thawed fish meat: European hake (Merluccius merluccius) as a possible model for white meat fish species Morphological and morphometrical discrimination of fresh and frozen/thawed fish meat: European-hake (Merluccius merluccius) as a possible model for white meat fish-species

Lara Tinacci ${ }^{1}$, Andrea Armani ${ }^{1 *}$, Alessandra Guidi ${ }^{1}$, Daniele Nucera ${ }^{2}$, Deborah Shvartzman, 7 Vincenzo Miragliotta ${ }^{1}$, Alessandra Coli ${ }^{1}$, Elisabetta Giannessi ${ }^{1}$, Maria Rita Stornelli ${ }^{1}$, Baldassare 8 Fronte $^{1}$, Francesco Di Iacovo ${ }^{1}$, Francesca Abramo ${ }^{1}$

${ }^{1}$ Department of Veterinary Sciences, University of Pisa, Viale delle Piagge 2, I-56124, Pisa, Italy.

${ }^{2}$ Department of Agriculture, Forest and Food Science, University of Turin, Largo Braccini 2, Grugliasco, Torino, Italy

\section{Corresponding author}

2 Andrea Armani, DVM- University of Pisa

3 Dept. Veterinary Sciences - Viale delle Piagge 2, 56124 - Pisa, Italy

Tel. $+39-050-2210207$

25 e-mail address: andrea.armani@unipi.it 


\section{Abstract}

The present study aimed at setting up a standard operating histological procedure to discriminate fresh from frozen-thawed fish products of the species Merluccius merluccius (European hake). A preliminary histological analysis of fresh M. merluccius muscle was performed to select the sampling site and highlight possible time-dependent tissue alterations during shelf-life. To set a suitable operational grid for discriminating the freezing process, morphological and morphometrical parameters were assessed on 90 muscle tissue samples collected from 30 fresh, 30 experimentally frozen at $-20^{\circ} \mathrm{C}$ and 30 Individual Quick Frozen (IQF) specimens of $M$. merluccius. Structural score, presence of freezing vacuoles, a number of vacuoles per field higher than 1.12 and the presence of interstitial serøproteinaceous material, which had achieved statistical significance in group comparisons were chosen as freezing markers. Accuracy and repeatability, assessed on the analysis of two independent operators (on-training and expert), showed high analytical specificity and sensitivity and a concordant diagnostic performance regardless the operators expertise. The grid was finally validated by a single blind test on 30 additional $M$. merluccius commercial products and allowed the allocation of all the samples to fresh or frozen status without inconclusive results. The method could be profitably applied against fraudulent adulteration practices.

\section{Keywords}

Seafood fraud, Freezing-thawing, histology, Merluccius merluccius, white meat fish species 


\section{Introduction}

Fish and seafood are widely accepted as essential components of a balanced diet thanks to their nutritional properties, in particular to their fatty acid composition (Domingo, Bocio, Falcó \& Llobet 2007). Based on lipid content and meat type, fish are commercially classified as fatty (i.e., salmon, herring, anchovy, sardine, and mackerel) or lean fish and as red or white meat fish (http://www.fao.org/wairdocs/tan/x5916e/x5916e01.htm); the latter includes both freshwater and marine, wild or farmed species characterized by low-medium fat content and the absence of dark fiber muscles (Ackman, 1989).

To date the whitefish market is one of the largest segment in the global seafood supply chain and at European level major products within this category are represented by cod (Gadus morhua), Alaska pollock (Gadus chalcogrammus) and hake species (Merluccius spp.) (EUMOFA, 2017). Hake demand has slightly increased over the last ten years compared to other species, reaching the highest value in 2014 and alone representing the $15 \%$ of the total ground fish originating from extra-EU countries. According to the report of the European Market Observatory for Fisheries and Aquaculture Products (EUMOFA, 2017), hake, that is reported as one of the highest ranked in terms of commercial value, is generally sold on the market as fresh or frozen. However, the peculiar chemical composition of fresh hake makes its shelf life limited due to enzymatic autolysis, lipid oxidation and microbial activities, which directly depends on post-mortem processing and storage (Ghaly, Dave, Budge \& Brooks, 2010).

Despite freezing is one of the most common method for seafood shelf-life extension and longterm preservation, the process is known to induce muscle structure changes and chemical modifications (protein denaturation, lipid oxidation, cell osmotic shrinkage and mechanical tissue damage caused by the intracellular and extracellular water crystallization and cellular dehydration), thus interfering with the overall organoleptic quality of the fish product (Zhu, Ramaswamy \& Simpson 2004; Venugopal., 2006; Burgaard, 2010; Gökoğlu \& Yerlikaya, 2015; Uddin, 2010). In 
addition, thawed fish is characterized by a higher perishability than chilled fish primarly due to microbiological spoilage. The microbial flora, only partially inactivated by the freezing process, is indeed positively affected by the increasing thawed tissue water activity (Pan \& Chow, 2004; Kolbe \& Kramer, 2007). All these aspects are main drivers for the European consumers' preference of fresh fish (Claret et al., 2012; Vanhonacker, Pieniak \& Verbeke, 2013; Reis et al., 2017). This preference greatly influence the market value of fresh and frozen fish. On the Italian market, the price of fresh hake is about $14.75 € / \mathrm{kg}$ while the frozen product is sold at $2.10 € / \mathrm{kg}$ (http://www.asapress.com/r-spesa/borsa170.html).

$$
\text { Information about the storage method involved in fish preservation represents one of the key }
$$
issues to guarantee the consumer's safety and awareness. Therefore, at the European level, Regulation EU No 1169/2011 and No 1379/2013 established that "information on the physical condition of the food or the specific treatment which it has undergone" must be reported on seafood labels. In the case of foods that have been frozen before sale and which are sold defrosted, the name of the food shall be accompanied by the designation 'defrosted'. However, for a consumer it is very hard to differentiate a fresh from a frozen-thawed fish on the basis of the organoleptic characteristics (Karoui, Thomas, \& Dufour, 2006), and deliberate substitution of frozen/thawed fish in place of fresh fish are recorded as common finding of fraudulent incidents (Uddin et al., 2005; Fasolato et al., 2008; Upton, 2015).

The occurrence of fraudulent substitutions leads to the setting of analytical methods for the discrimination of frozen-thawed and fresh fish. This has been accomplished by means of morphological, physiological, chemical and physical parameters (Duflos, Le Fur, Mulak, Becel \& Malle., 2002; Uddin, 2010). However, the reliability of these methods is limited in case of fish with a long shelf life (Duflos et al., 2002) and in presence of processed products such as skinned and filleted fish (Hassoun \& Karoui, 2017). More recently, alternative physical methods based on frontface fluorescence, near infrared spectroscopy, solid-phase gas chromatography and mass spectrometry, have been proposed as non-destructive methods for fresh-frozen product 
discrimination (Karoui et al., 2006; Uddin 2010, Fasolato et al., 2012, Leduc et al., 2012, Zhu et al. 2013, Ottavian, Fasolato, Facco, \& Barolo, 2013). Although all methods have been shown to be effective for the analysis of whole and filleted products they require a large set of reference samples for the assay validation and the development of calibration models for each species (Ottavian et al., 2013; Hassoun \& Karoui, 2017).

An alternative method for discriminating fresh and frozen/thawed products is based on the recognition of histological structural changes (empty vacuolar spaces) induced by freezing (Love, 1958; Simeonidou, Govaris \& Vareltzis, 1997; Sigurgisladóttir S, Ingvarsdóttir H, Torrissen OJ, Cardinal M, Hafsteinsson, 2000; Alizadeh, Chapleau, De Lamballerie, \& Le-Bail, 2007; Alizadeh, Chapleau, De Lamballerie, \& Le-Bail, 2009). This approach, firstly proposed as a discriminating method for the common carp (Cyprinus carpio L.) (Pavlov, Dimitrov, Penchev \& Georgiev, 2008), has been recently applied to other species characterized by different muscular composition and fat content: gilthead (Sparus aurata), red mullet (Mullus barbatus), swordfish (Xiphias gladius), bonito (Sarda sarda), salmon (Salmo salar), turbot (Psetta maxima), albacore (Thunnus alalunga), little tunny (Euthynnus alletteratus), rainbow trout (Oncorhyncus mykiss) and anchovy (Engraulis encrasicolus) (Bozzetta et al., 2012; Richelmi et al., 2013; Popelka, Nagy, Pipová, Marcinčák, \& Lenhardt, L., 2014, Meistro et al., 2016). Despite the high accuracy of these methods in the detection of the freezing process, the presence of unspecific microscopic alterations reduced the assay accuracy and precision leading to "non-conclusive results". In addition, possible microscopic alterations eventually occurring within the product's shelf-life were not assessed.

The aim of the present study was to contribute in setting up a standard operating histological procedure that enables to discriminate fresh from frozen-thawed fish products for $M$. merluccius (European hake). Preliminary objectives of this study were to investigate the histological pattern of fresh $M$. merluccius muscle tissue for selecting the most appropriate sampling site and to assess possible time dependent tissue modifications during shelf-life. Secondly, histological parameters were recorded for the differentiation of fresh and frozen muscle tissue of $M$. merluccius. Finally, an 
operational protocol was set up and tested on a subset of randomly selected previously examined samples and then validated by a single-blind control procedure on commercial samples.

\section{Materials and Methods}

\subsection{Tissue histology of fresh M. merluccius muscle for sampling site selection}

2.1.1 Specimens collection and processing. Eight fresh medium size (200-300 g) whole M. merluccius (caught within the previous 24 hours) were collected at a local fish market. Two cm long fresh muscle samples were obtained from the left side of each fish, from three different anatomical sites: a) the lateral line, b) dorsal muscle next to the column and distant from the lateral line and c) ventral muscle posterior to the anal opening; (Fig. 1). Samples were either promptly fixed in a $10 \%$ buffered formalin solution $(\mathrm{pH}$ 7.4) for paraffin embedding or cryo-protected with $30 \%$ sucrose for cryo-sectioning. Tissue processing of formalin fixed samples was performed in a controlled automatic processor (Shandon TP 1020; Leika, Milan, Italy) and paraffin embedding was accomplished to obtain transversal sections of the muscle fibers. Five $\mu \mathrm{m}$ thick sections were stained with hematoxylin and eosin (H\&E) under standard protocol. Cryo-sectioned samples were stained with Oil Red O to evaluate the lipid distribution within samples.

\subsection{Assessment of histological time-dependent tissue modification on fresh M. merluccius}

To avoid the misinterpretation of hypothetical shelf-life time-dependent alterations as thawing modifications, other 15 whole fresh specimens of medium size (200-300 g; caught within the previous 24 hours) obtained at a local fish market, were included in this study. Of these, 5 were sampled within 24 hours (24H group); 5 were sampled after 48 hours of conservation at $4{ }^{\circ} \mathrm{C}(72 \mathrm{H}$ group) and the last 5 specimens, after an additional conservation at $4{ }^{\circ} \mathrm{C}$ for further 48 hours $(120 \mathrm{H}$ group). The samples, all collected from the dorsal muscle, were processed as previously described and all alterations observed were recorded. The observations were conducted within 10 consecutive fields at 20x magnification, each field corresponding to $567-073 \mu \mathrm{m}^{2}$. Areas occupied by timedependent tissue modifications were recorded on H\&E stained sections using a light microscope 
(Nikon, Eclipse 80i) connected to a personal computer via a Nikon digital camera (Digital Sight DS-U1) and measurements were carried out with the NIS-Elements $\mathrm{Br}$ accompanying software.

\subsection{Histological evaluation of fresh and frozen M. merluccius}

2.3.1 Sampling. Histological evaluation was performed on a total of 90 samples as follows. Thirty fresh M. merluccius (caught within the previous 24 hours) of about 200-300 g weight, were collected at a local fish market. A muscle punch from the dorsal muscle was promptly fixed in a $10 \%$ formalin solution (fresh $M$. merluccius, F_MM). The remaining fish were frozen in a conventional laboratory freezer at $-20{ }^{\circ} \mathrm{C}$ for 15 days; then, after controlled thawing $\left(4{ }^{\circ} \mathrm{C}\right.$ for 12 hours), 30 new tissue samples were collected in the contralateral area, symmetrically to the first sampling site (CF_MM, conventionally frozen M. merluccius). Moreover, other 30 M. merluccius fish that had undergone an Individual Quick Freezing (IQF)_process, which is usually performed in an air-blast tunnel at $-35^{\circ} \mathrm{C}$ to $-45^{\circ} \mathrm{C}$ for $1 \mathrm{~h}$ to $3 \mathrm{~h}$ and a different speed according to the fish size (200g to $400 \mathrm{~g}$ (Venugopal, 2006), were purchased and sampled after controlled thawing (IQF_MM, commercially frozen M. merluccius). After processing, sectioning and H\&E staining (see section 2.1.1) morphological and morphometric parameters were recorded for the differentiation of fresh and frozen muscle tissue of M. merluccius.

2.3.2 Morphology. After a preliminary screening of histological slides, the following parameters were selected for morphological assessment: a) the overall muscle structural organization, b) the presence of freezing vacuoles defined as polygonal spaces with smooth angles within the muscle myofiber and c) the presence of interstitial proteinaceous material, defined as a slightly granular basophilic material accumulated in the interstitium between myofibers. These parameters were scored on four randomly selected areas and: a) overall structural organization (assessed at a 10x magnification) was scored as $0=$ fully destructured muscle, as $1=$ partially $(<50 \%)$ destructured muscle , 2= well preserved muscle ; b) myofiber vacuolization (assessed at a 20x magnification) was recorded as $0=$ absence and $1=$ presence; $c$ ) interstitial proteinaceous material, (observed at a 10x magnification) was scored as $0=$ absence and $1=$ presence. 
2.3.3 Morphometry. Four hot spot areas from samples that scored either 1 or 2 at the above mentioned parameter "a)" were selected at low power and measurements performed at 20x magnification within a predetermined field. Total number of vacuoles in the four selected fields, number of vacuoles per fields, mean vacuole size, mean size of myofibers containing vacuoles and percentage of the myofiber occupied by vacuoles were recorded. Analyzes were performed on H\&E stained sections as mentioned above (2.2).

\subsection{Statistical analysis}

2.4.1 Shelf life test. A paired-sample t-test was used to assess the difference in lysis surface between samples kept for 24 hours and those kept for 72 and 120 hours. Results were considered significant when $\mathrm{p}<0.05$.

\subsubsection{Selection of the parameters for discrimination of fresh and frozen-thawed fish muscle.}

Different statistical tests were applied as follows. The organization of the muscle structure (score) was analysed by comparing the score distribution (from 0 to 2) within the three groups using the Kruskal-Wallis test; if overall significance was observed, further differences among groups were assessed using the Mann-Withney $\mathrm{U}$ test with k-1 comparisons ( $\mathrm{k}$ is the total number of examined groups). The presence (1) or absence (0) of vacuoles and sereproteinaceous material was evaluated by the chi-squared test to compare differences of frequency of positive samples (presence of vacuoles and interstitial sereproteinaceous material) within the three groups (F_MM, CF_MM, IQF_MM). The same test was used to compare the effect of freezing (presence of vacuoles and sereproteinaceous material) by the comparison of both CF_MM and IQF_MM, evaluated together, against group F_MM. Morphometrical parameters (number of vacuoles per fields, mean vacuole size, mean size of myofibers containing the vacuoles and percentage of myofiber occupied by vacuoles) were investigated using the ANOVA test. When a significant result was obtained a posthoc Dunnet test was performed. For all the analyses, significant results were those associated with $\mathrm{p}<0.05$. The parameters that were confirmed as significant were used to set up the final protocol. In case of morphometric parameters, a cut-off value was established. In particular, the number of 
vacuoles per field parameter was selected to define a cut-off value for the discrimination of fresh and frozen products. The cut-off limit for the discrimination of freezing was determined using the 95\% Confidence Interval (95\% C.I.) calculated on the mean number of vacuoles per area in the F_MM group $(95 \%$ C.I. $=0-1.12)$ and using its upper level, considering that the two other groups were characterized by means of $2.24-4.47\left(\mathrm{CF} \_\mathrm{MM}\right)$ and 6.77-9.95 (IQF_MM) with 95\% C.I. Thus, all those samples showing a number of vacuoles per field equal or below 1.12 were considered fresh, conversely, all the samples showing a higher value were classified as frozen.

\subsection{Operational protocol and assessment of the role of the operators}

2.5.1. Operational protocol. An evaluation grid was designed using morphological and morphometric parameters and the cut-off level established by statistical analyses (Fig. 2). Then, it was presented to the operators concurrently to the histological sections to issue a judgment of freezing expressed as Positive (frozen) and Negative (fresh).

2.5.2. Assessment of the role of the operators. Two independent operators, a student (Operator 1: on-training) and a pathologist (Operator 2: expert), were asked to use the operational protocol to reclassify 50 out of the 90 samples selected by the Stat Trek random number generator (http://stattrek.com/statistics/random-number-generator.aspx). Randomly selected samples were 17 fresh and 33 frozen (18 CF_MM, and 15 IQF_MM). Sensitivity and specificity achieved by the two operators were calculated using contingency table analysis. The level of concordance between Operator 1 and 2 was evaluated with the Cohen statistical index $\mathrm{k}$. With a $\mathrm{k}$ Cohen index $>80 \%$ satisfactory concordance was achieved, while full concordance was defined as a $100 \% \mathrm{k}$ Cohen index. For these analyses EPI6 software for windows was used (Dean et al., 1994).

\subsection{Final Validation}

Thirty additional commercial fish, belonging to both fresh (caught within the 24hours; 13 specimens) and IQF frozen (17 specimens), were collected and processed as described in section 2.1.1. These samples were presented to the operators without any indication about their origin (single-blind control procedure). The judgment (fresh or frozen) was issued through the analysis of 
three histological sections for each sample. In particular, the operators were asked to issue the final judgment on the basis of the result obtained on at least two out of the three sections analyzed for each sample according to the evaluation grid developed in this study (Fig. 2).

\section{Results}

\subsection{Tissue histology of fresh M. merluccius muscle for sampling site selection}

Myofibers of fresh $M$. merluccius were always arranged in fascicles surrounded by connective tissue. Two different myofiber types were identified at the H\&E staining: large polygonal fibers whose cytoplasm was packed with myofibrils and small myofibers whose cytoplasm often showed several small round empty spaces (Fig. 3a). Oil Red O staining showed that the empty spaces found in $\mathrm{H} \& \mathrm{E}$ stained sections were lipid droplets (Fig. 3b). Fascicles containing lipid droplets were found lying between the skin and the underneath muscle in all samples collected from the lateral line (100\% of the specimen), in $25 \%$ of samples collected from the dorsal muscle and in half of the samples obtained from the ventral muscle (50\%). In samples from the lateral line and the ventral area they were also found within the deep muscle tissue. Thus, the dorsal area was selected as sampling site.

\subsection{Assessment of histological time-dependent tissue modifications on fresh M. Merluccius}

The assessment of fresh $M$. merluccius at different shelf-life time points revealed histological focal areas of either swollen (Fig. 4a) and shrunken-fragmented (Fig. 4b) eosinophilic lytic myofibers. On the ten total fields observed at $20 \mathrm{X}$ of magnification, shrunken-fragmented myofibers were observed in 2 to 6 fields in the samples at $24 \mathrm{~h}$ of storage (mean $=3.2$ ); 2 to 4 at $72 \mathrm{~h}$ $(m e a n=3)$ and 0 to 6 at $120 \mathrm{~h}($ mean=3.4). Significant differences were not found when comparing the size of lytic areas over different shelf life samples (data not shown).

\subsection{Histological evaluation of fresh and frozen M. merluccius}

3.3.1. Morphological assessment. The preliminary screening of histological slides showed the presence of:

1. Different degree of muscle destructuration; 
2. Freezing vacuoles (Fig. 5a) recognized for their squared or polygonal shape, smooth margin and empty space or space filled with a slightly basophilic material;

3. Seroproteinaceous-Proteinaceous material in the interstitial space (Fig. 5b).

4. Myofiber empty spaces of irregular angular shape (Fig. 5c) or as thin short empty fractures (Fig. 5d). These alterations were observed occasionally in both fresh (F_MM) and frozen (CF_MM and IQF_MM) tissues and were considered as artefactual findings produced by tissue processing;

5. Either swollen and shrunken-fragmented eosinophilic myofibers (lytic fibers) were seen, without significance differences, in all groups.

Considering that our goal was to select "changes" related to the freezing process, artefactual findings (point 4) and lytic myofibers (point 5) were not included as parameters to be used for the discrimination of fresh and frozen-thawed muscle tissue.

As regards the other selected parameters results are summarized in Table 1 and reported in detail in Table 1SM. The structural organization of the muscular component was generally well maintained in F_MM samples. No vacuolar alterations similar to freezing vacuoles were recorded except for three samples in which only 1 intracellular vacuole in 3 out of 4 fields of observation was observed. In CF_MM partial or full muscle tissue destructuration prevailed. In the presence of fully destructured score (0) the freezing vacuoles were broken and uncomplete (not delimited) and surrounded by released sereproteinaceous material; thus the samples were not morphometrically evaluable. In IQF_MM the structural organization of the muscular component was generally well maintained. Noteworthy, freezing vacuoles of regular shape were homogeneously distributed within the single myofibers, while vacuoles in the CF_MM were randomly and not homogeneously scattered throughout the muscle fibers.

Kruskall-Wallis test for overall structural organization showed high statistically significant differences $(\chi 2=44.68 \mathrm{p}<0.001)$ between the tree groups. When Mann-Whitney test was performed CF_MM (mean 0.9) was different from either F_MM (mean 1.9) showing $z=-4.83, p<0.001$ or 
IQF_MM (mean 2.0), with $\mathrm{z}=-5.05, \mathrm{p}<0.001$. On the contrary, no significant differences were found on structural scores between F_MM and IQF_MM groups. Chi-squared test showed a significant effect of freezing for frequency of vacuoles $(\chi 2=69.1 \mathrm{p}<0.001)$ and interstitial proteinaceous material $(\chi 2=80.3 \mathrm{p}<0.001)$ when all groups were included in the statistical analysis. When pairwise comparisons were made, CF_MM was not statistically different from IQF_MM. By aggregating data from frozen samples (CF_MM + IQF_MM) and comparing them against data of fresh tissues (F_MM) results were again statistically significant for both the parameters (presence of vacuoles: $\chi 2=64.9, p<0.001$; presence of serøproteinaceous material: $\chi 2=77.0, p<0.001)$.

3.3.2. Morphometrical assessment. As reported in section 2.3.3, the assessment of morphometrical parameters (number of vacuoles per fields, mean vacuole size, mean size of myofibers containing the vacuoles and percentage of the myofiber occupied by vacuoles) was performed only on samples that scored either 1 or 2 as regards muscle organization (Table 1). In particular, 3 F_MM samples, 18 CF_MM and all the IQF_MM samples were assessed.

Mean number of vacuoles per field progressively increased from F_MM (0.5 per field, ranging from 0.25 to 0.75$)$ to CF_MM (3.50 per field, ranging from 1 to 8.75) and IQF_MM (8.3 per field, ranging from 3.75 to 19$)$. Similarly, mean percentage of the myofiber occupied by vacuoles was $11 \%$ in F_MM, 22.1\% in CF_MM and 31\% in IQF_MM (Table 1SM).

The mean values of the two quantitative parameters (number of vacuoles per field and percentage of the myofiber occupied by vacuoles), investigated using the ANOVA test, were confirmed significantly different across groups $(\mathrm{F}=64.3, \mathrm{p}<0.001$ for vacuoles per field and $\mathrm{F}=257.8 \mathrm{p}<0.001$ for percentage of myofiber occupied). When Dunnett test was performed, the difference was statistically significant between F_MM and CF_MM ( $\mathrm{p}<0.001)$ as well as F_MM and IQF_MM $(\mathrm{p}<0.001)$ for both parameters. Thus, they were confirmed as applicable indices for the discrimination between fresh and frozen products.

\subsection{Operational protocol and assessment of the role of the operators}


3.4.1. Operational protocol. Above mentioned parameters that achieved statistical significance in group comparisons were included in the final operational grid for the discrimination of fresh and frozen-thawed M. Merluccius (Fig. 2). The operators were asked to use the grid to reclassify a randomly selected blind set of previously examined samples $(n=50)$; samples that achieved a cutto frozen status only in presence of interstitial serøproteinaceous material.

3.4.2. Assessment of the role of the operators: reliability assessment. Both operators assigned all (95\% C.I.: $85 \%-100 \%)$ and $94 \%$ specificity (95\% C.I.: $71 \%-100 \%)$ while Operator 2 (expert) showed $97 \%$ sensitivity (95\% C.I: $84 \%-100 \%)$ and $100 \%$ specificity (95\% C.I.: $80 \%-100 \%)$. The Cohen index used to evaluate the degree of agreement between the two classifications was $k=91 \%$, p $<0.01$ (95\% C.I.:79\%-100\%). This confirmed a significant analytical concordance between the operators. each sample.

\subsection{Final validation of the operational procedure}

Both the operators assigned all the 30 additional commercial fish samples (13 fresh and 17 IQF) to the correct category and the scores attributed to the three sections were analogous, confirming a substantial structural homogeneity between the different portions of the tissue punch collected from

\section{Discussion}

\subsection{Tissue histology of fresh M. merluccius muscle for sampling site selection}

To evaluate the anatomical distribution of muscle fibers and to obtain homogeneous data for statistical comparisons, a specific anatomical sampling site was identified by a preliminary analysis of $M$. merluccius tissue histology. This preliminary step also aimed at recognizing the presence of any vacuolar shaped intracellular space that would resemble the myofiber vacuolization reported as the main change associated with freezing (Ayala et al., 2005; Bozzetta et al., 2012; Meistro et al., 
2016). Previous studies, conducted on 84 marine species, have shown different distribution and variable percentages of white and red muscle fibers (Greek-Walker \& Pull, 1975). Regardless of the species, the dorsal muscles are predominantly composed of white fibers while the red ones, if present, are exclusively localized in the most superficial portion (Johnston, 1981; Greek-Walker \& Pull, 1975). This pattern was confirmed in this study also for the specie M. merluccius for which a precise anatomical distribution of the two muscle fiber types had not been described before. Therefore, even though lipid vacuoles can be easily differentiated from freezing vacuoles for their shape and size (small, perfectly round, optically empty on H\&E stained sections) the dorsal area was chosen as the elective site in this study as more homogeneous by a structural point of view. Thus, this area might in fact represent a "species-independent" reference sampling site for the analysis of white fish species even by not specifically trained operators.

\subsection{Assessment of histological time-dependent tissue modifications on fresh M. merluccius}

The possible onset of tissue modifications related to fish spoilage within the expected shelf life of the product, that are generally due to the combination of enzymatic autolysis oxidation and microbial growth (Ghaly et al., 2010; George, Van Wettere, Michaels, Crain, \& Lewbart, 2016), was also considered in the preliminary assessment. Since myofiber vacuoles are reported as the main change associated with freezing, the analysis was focused on the detection of the possible

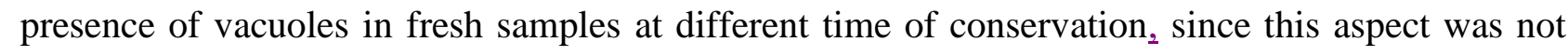
considered in the previous studies (Bozzetta et al., 2012; Popelka et al., 2014; Meistro et al., 2016). In this respect, George et al. (2016), in a study about histopathologic evaluation of post mortem changes in fresh water fish species preserved in several storage conditions (room temperature, refrigeration, freezing) and at different sampling intervals (4, 24, 48 hours), reported the onset of mild to evident tissue alterations (cellular oedema/swelling) subsequent to autolytic phenomena in all storage conditions. Conversely, the vacuolar lesions recorded were all exclusively associated to freezing-thawing processes. Accordingly, in the present study no vacuolar changes were found in samples at 24, 72 and 120 hours (kept at $4{ }^{\circ} \mathrm{C}$ ) analyzed in the study. On the contrary scattered 
areas of swollen and shrunken-fragmented eosinophilic fibers were recorded. These alterations were similar to those described by Sigurgisladottir et al. (2000) in frozen salmon (Salmo salar) and by Popelka et al. (2014) in rainbow trout (Oncorhyncus mykiss), likely due to autolytic enzymes which are known to be the main responsible for the post mortem tissue softening (Ahmed, Donkor, Street, \& Vasiljevic, 2015).

\subsection{Histological evaluation of fresh and frozen M. merluccius}

Once determined that small round vacuoles present in fresh samples were lipid filled and that either swollen and shrunken-fragmented eosinophilic fibers were likely a consequence of autolysis, the further assessment of fresh $v s$ frozen-thawed samples aimed at identifying peculiar parameters related to freezing for being included in the final operative analytical protocol.

Tissue de-structuring was the first parameter included in the list of the recorded alterations: this finding was primarily observed in $\mathrm{CF}_{-} \mathrm{MM}$ and not in IQF_MM samples in which muscle organization was generally well maintained. In this respect, the use of a slow freezing technique might have led to the onset of osmotic phenomena, as already reviewed by Pham (2008) and Kiani and Sun (2011), leading to morphological alterations such as dehydration and shrinkage. Moreover, it is known that the slow rate of temperature decrease enhances the formation of large extracellular ice crystals (Kiani \& Sun, 2011). All these phenomena predispose to the breaking of the cell membranes during the thawing procedure (Pham, 2008). On the contrary, a fast-freezing technique, as the IQF technology, generally produces a more uniform intracellular and extracellular water crystallization (Pham, 2008). This aspect was further confirmed in this study by the absence of significant differences between the structural scores of F_MM and IQF_MM. Therefore, a tissue structure score might be proposed as parameter to estimate the quality of the freezing process during product' shelf life. In fact, the high quality of frozen seafood may be lost either by interruption of the cold chain during transportation (Gormley, Walshe, Hussey, \& Butler, 2002) or by non-industrial freezing improperly applied by wholesalers to slow down the tissue spoilage mechanisms of unsold fresh products (Bozzetta et al., 2012). 
The second and third listed parameters (freezing vacuoles and sereproteinaceous material) were found to be significantly freezing-dependent. Non-homogeneous distribution of freezing vacuoles in $\mathrm{CF} \_\mathrm{MM}$ samples compared to the homogeneous pattern observed in IQF_MM, was consistent with findings previously described by Ayala et al. (2005). Also the presence of interstitial proteinaceous material in the interstitium between myofibers in CF_MM samples, was plausibly due to the formation of ice macrocrystals induced by slow freezing process for the tissue deformation and the impairment of cell membrane integrity (Pham, 2008; Alizadeh et al., 2007).

\subsection{Operational protocol and assessment of the role of the operators and final validation}

The high specificity and sensitivity of the operational protocol in this study was confirmed by the

reliability assessment of the two operators. There was a low probability of false positive and false overlooked by the trainee but not experienced operator. contingency table was related to the operator's experience since it was hypothesized that an expert operator may consider a minimal vacuolar change as a freezing vacuole while these may be The application of the cut-off threshold obtained from the statistical analysis on the quantitative parameter vacuole per field, allows the allocation of all the samples to the fresh or frozen status thus avoiding inconclusive results.

\section{Conclusions}

Even though several analytical methods can help in the identification of frozen products sold as fresh, these techniques are often cost, and reagent demanding and require highly skilled operators. Therefore, industry and official authorities are interested in convenience, non-destructive, noninvasive and cost-effective methods. In the present study the use of histology as suitable analytical tool to prevent fraudulent substitutions of fresh with frozen-thawed fish, was confirmed. The selected histological parameters and the final operative protocol applied to the European hake (M. merluccius), may represent a reliable and cost-effective procedure to be proposed for the analysis of white fish category. Further experiments are however needed to confirm the possibility of applying 
the protocol to different species. The method can be also applied to verify both the quality of the freezing process and the correct maintenance of the cold chain of frozen products during transport and storage phases before sale. Besides the scientific evidences offered by the study possible expected outcomes are linked to the increasing reliance, transparency and trust between diverse actors along the chain that may enhance market competitiveness as well as consumers wellness.

\section{Acknowledgments}

The authors wish to thank the Quality Office of UNICOOP Firenze for its contribution to the research activities, which were carried out in the framework of a survey aimed at assessing the overall quality of seafood products.

\section{Funding}

This project has been funded by the University of Pisa (Project PRA_2017_24).

\section{Figures captions}

Fig. 1. Anatomical position of the three sampling sites of muscular tissue (tissue punch length = $2 \mathrm{~cm}$ ) evaluated in the study on Merluccius merluccius exemplar (size = $250 \mathrm{~g}$ ). a) lateral line, b) dorsal muscle, c) ventral muscle.

Fig. 2. Evaluation grid proposed to the operators for the analysis of to the histological sections. *Score structure: 2 = well preserved muscle, $1=$ partially destructured muscle, $0=$ fully destructured muscle. Final judgement: $\mathrm{N}=$ negative (fresh product), $\mathrm{P}=$ positive (frozen product).

Fig. 3. Detail of a histological section of Merluccius merluccius dorsal muscle. a) Small myofibers with several small round empty spaces within the cytoplasm that are grouped in a fascicle (asterisk) laying between the superficial connective tissue (arrowhead) and underlying large polygonal muscle fibers (H\&E staining, bar $200 \mu \mathrm{m}$ ). b) Small round spaces within the cytoplasm of the small myofibers laying beneath the connective tissue (arrowhead) red stained with Oil Red $\mathrm{O}$ for lipids (asterisk) (bar $100 \mu \mathrm{m})$.

Fig. 4. Histology of Merluccius merluccius muscle at different shelf-life time. a) Scattered multifocal homogeneously swollen deep eosinophilic myofibers (arrowheads) at 72h shelf-life and b) grouped shrunken and fragmented lytic myofibers (arrows) at 120h shelf-life (H\&E stain, bar $100 \mu \mathrm{m})$.

Fig. 5. Histology of Merluccius merluccius muscle with different myofiber alterations. a) Squared and round freezing vacuoles (asterisk) with empty spaces or spaces containing slightly basophilic material, b) sereproteinaceous material (arrowheads) in the interstitial space among myofibers containing freezing vacuoles, c) myofiber empty spaces of irregular angular shape (arrows) not related to freezing and d) thin short empty fractures within myofibers not related to freezing (H\&E stain, bar $100 \mu \mathrm{m})$. 


\section{References}

451

452

453

454

455

456

457

458

459

460

461

462

463

464

465

466

467

468

469

470

471

472

473

474

475

476

477

478

479

480

481

482

Ackmann RG (1989) Nutritional composition of fats in seafoods. Progress in Food \& Nutrition Science, 13(3-4),161-289

Ahmed, Z., Donkor, O., Street, W. A., \& Vasiljevic, T. (2015). Calpains-and cathepsins-induced myofibrillar changes in post-mortem fish: Impact on structural softening and release of bioactive peptides. Trends in Food Science \& Technology, 45(1), 130-146.

Alizadeh, E., Chapleau, N., De Lamballerie, M., \& Le-Bail, A. (2007). Effect of different freezing processes on the microstructure of Atlantic salmon (Salmo salar) fillets. Innovative Food Science \& Emerging Technologies, 8(4), 493-499.

Alizadeh, E., Chapleau, N., de-Lamballerie, M., \& Le-Bail, A. (2009). Impact of freezing process on salt diffusivity of seafood: application to salmon (Salmo salar) using conventional and pressure shift freezing. Food and bioprocess technology, 2(3), 257-262.

Ayala, M. D., Albors, O. L., Blanco, A., Alcázar, A. G., Abellán, E., Zarzosa, G. R., \& Gil, F. (2005). Structural and ultrastructural changes on muscle tissue of sea bass, Dicentrarchus labrax L., after cooking and freezing. Aquaculture, 250(1), 215-231.

Bozzetta, E., Pezzolato, M., Cencetti, E., Varello, K., Abramo, F., Mutinelli, F., Ingravalle, F., \& Teneggi, E. (2012). Histology as a valid and reliable tool to differentiate fresh from frozen-thawed fish. Journal of Food Protection, 75(8), 1536-1541.

Burgaard, M. G. (2010). Effect of frozen storage temperature on quality-related changes in fish muscle: Changes in physical, chemical and biochemical quality indicators during short- and long-term storage. Kgs. Lyngby, Denmark: Technical University of Denmark (DTU). Available at www.food.dtu.dk

Claret, A., Guerrero, L., Aguirre, E., Rincón, L., Hernández, M. D., Martínez, I., Peleteiro J. B., Grau A., Rodríguez-Rodríguez, C. (2012). Consumer preferences for sea fish using conjoint analysis: Exploratory study of the importance of country of origin, obtaining method, storage conditions and purchasing price. Food Quality and Preference, 26(2), 259-266.

Dean AG, Dean JA, Coulombier D, Brendel KA, Smith DC, Burton AH, et al. (1994). Epi info Ver 6: A word processing, database and statistics programme for epidemiology on microcomputers. Atlanta, Georgia, USA: Centre for Disease Control and Prevention. Available at: http://www.cdc.gov/epiinfo/Epi6/ei6.htm.

Domingo, J. L., Bocio, A., Falcó, G., \& Llobet, J. M. (2007). Benefits and risks of fish consumption: Part I. A quantitative analysis of the intake of omega-3 fatty acids and chemical contaminants. Toxicology, 230(2), 219-226. 

differentiating between fresh and frozen-thawed fish or fillets. Journal of the Science of Food and Agriculture, 82(12), 1341-1

EUMOFA, 2017. http://www.eumofa.eu/documents/20178/108446/The+EU+fish+market+2017.pdf

Fasolato, L., Balzan, S., Riovanto, R., Berzaghi, P., Mirisola, M., Ferlito, J. C., Serva, L., Benozzo, F., Passera, R., Tepedino, V., \& Novelli, E. (2012). Comparison of visible and near-infrared reflectance spectroscopy to authenticate fresh and frozen-thawed swordfish (Xiphias gladius L). Journal of Aquatic 490 Food product Technology, 21(5), 493-507.

Fasolato, L., Mirisola, M., Tepedino, G., Balzan, S., Arcangeli, G., Rosteghin, M., Corrain, C., 492 Manfrin, A., \& Berzaghi, P. (2008). Mai più decongelato per fresco. Dossier Eurofishmarket, Available at https://www.eurofishmarket.it/b2b/decongelato.pdf

George, J., Van Wettere, A. J., Michaels, B. B., Crain, D., \& Lewbart, G. A. (2016). Histopathologic 495 evaluation of postmortem autolytic changes in bluegill (Lepomis macrohirus) and crappie (Pomoxis 496 anularis) at varied time intervals and storage temperatures. PeerJ, 4, e1943.

Ghaly, A. E., Dave, D., Budge, S., \& Brooks, M. S. (2010). Fish spoilage mechanisms and 498 preservation techniques. American Journal of Applied Sciences, 7(7), 859.

Gökoğlu, N., \& Yerlikaya, P. (2015) Chapter 7: Freezing technology In: Gökoğlu, N \& Yerlikaya P. (Eds.). Seafood chilling, refrigeration and freezing: Science and Technology. Wiley \& sons, 2015 ( $1^{\text {st }}$ ed $)$ (pp.163-185).

Gormley, R., Walshe, T., Hussey, K., \& Butler, F. (2002). The effect of fluctuating vs. constant frozen storage temperature regimes on some quality parameters of selected food products. Lebensmittelwissenschaft Und-technologie-food Science and Technology, 35(2), 190e200.

Greek- Walker, M., \& Pull, G. A. (1975). A survey of red and white muscle in marine fish. Journal 506 of Fish Biology, 7(3), 295-300.

Hassoun, A., \& Karoui, R. (2017). Quality evaluation of fish and other seafood by traditional and nondestructive instrumental methods: Advantages and limitations, Critical Reviews in Food Science and Nutrition, 57(9), 1976-1998, DOI: 10.1080/10408398.2015.1047926.

Johnston IA. (1981). Structure and function of fish muscles. Symp. zaal. Sac. Land. 48. 71-113

Karoui, R., Thomas, E., \& Dufour, E. (2006). Utilisation of a rapid technique based on front-face fluorescence spectroscopy for differentiating between fresh and frozen-thawed fish fillets. Food Research International, 39, 349-355.

Kiani, H., \& Sun, D. W. (2011). Water crystallization and its importance to freezing of foods: A review. Trends in Food Science \& Technology, 22(8), 407-426.

Kolbe, E., \& Kramer, D. (2007). Planning for seafood freezing. Alaska sea Grant College Program, Cooper Publishing, Alaska. pp.112

Leduc, F., Krzewinski, F., Le Fur, B., N'Guessan, A., Malle, P., Kol, O., \& Duflos, G. (2012). Differentiation of fresh and frozen/thawed fish, European sea bass (Dicentrarchus labrax), gilthead 
seabream (Sparus aurata), cod (Gadus morhua) and salmon (Salmo salar), using volatile compounds by SPME/GC/MS. Journal of the Science of Food and Agriculture, 92, 2560-2568

Love R.M. (1958). The expresible fluid of fish fillets IX. Other types of cell damage caused by freezing. 3. Sci. Food Agric. 9:262-268, 1958.

Meistro, S., Pezzolato, M., Muscolino, D., Giarratana, F., Baioni, E., Panebianco, A., \& Bozzetta, E. (2016). Histology as a Valid Tool to Differentiate Fresh from Frozen-Thawed Marinated Fish. Journal of Food Protection, 79(8), 1457-1459.

Ottavian, M., Fasolato, L., Facco, P., \& Barolo, M. (2013). Foodstuff authentication from spectral data: toward a species-independent discrimination between fresh and frozen-thawed fish samples. Journal of Food Engineering, 119, 765-775.

Pan, B., \& Chow, C.J. (2004). Freezing secondary seafood products. In: Nollet, L.M..L, \& Toldra F. 531 (Eds.) Handbook of Frozen products. CRC Press, Boca Raton (pp. 325-339)

Pavlov, A., Dimitrov, D., Penchev, G., \& Georgiev, L. (2008). Structural changes in common carp 533 (Cyprinus carpio L.) fish meat during freezing. Bulgarian Journal of Veterinary Medicine, 11(2), 131534 136.

Pham, Q. T. (2008). Advances in food freezing/thawing/freeze concentration modelling and 536 techniques. Japan Journal of Food Engineering, 9(1), 21-32.

Popelka, P., Nagy, J., Pipová, M., Marcinčák, S., \& Lenhardt, L. (2014). Comparison of chemical, microbiological and histological changes in fresh, frozen and double frozen rainbow trout (Oncorhynchus mykiss). Acta Veterinaria Brno, 83(2), 157-161.

Regulation (EU) No 1169/2011 of the European Parliament and of the Council of 25 October 2011 on the provision of food information to consumers, amending Regulations (EC) No 1924/2006 and (EC) No 1925/2006 of the European Parliament and of the Council, and repealing Commission Directive 87/250/EEC, Council Directive 90/496/EEC, Commission Directive 1999/10/EC, Directive 2000/13/EC of the European Parliament and of the Council, Commission Directives 2002/67/EC and 2008/5/EC and Commission Regulation (EC) No 608/ 2004. Official Journal of the European Union, L 304.

Regulation (EU) No 1379/2013 of the European Parliament and of the Council of 11 December 2013 on the common organisation of the markets in fishery and aquaculture products, amending Council Regulations (EC) No 1184/2006 and (EC) No 1224/2009 and repealing Council Regulation (EC) No 104/2000. Official Journal of the European Union, L 354.

Reis, M. M., Martinez, E., Saitua, E., Rodriguez, R., Perez, I., \& Olabarrieta, I. (2017). Non-invasive differentiation between fresh and frozen/thawed tuna fillets using near infrared spectroscopy (Vis-NIRS). LWT-Food Science and Technology, 78, 129-137.

Richelmi, G. B., Pezzolato, M., Gili, S., Gallina, S., Decastelli, L., Tarasco, R., Abete, M.C., Ingravalle, F., Serracca, L., Pavino, D., Vivaldi, B., Riina, M.V., Acutis, P.L., Prearo, M., Caramelli, M., \& Bozzetta E. (2013). Pilot project to set up a control programme on fishery products. Italian Journal of Food Safety, 2(2), 25. 
Sigurgisladóttir S, Ingvarsdóttir, H., Torrissen, O.J., Cardinal, M., \& Hafsteinsson, H. (2000) Effects of freezing/thawing on the microstructure and the texture of smoked Atlantic salmon (Salmo salar). Food Research International, 33, 857-865

Simeonidou S, Govaris A, Vareltzis K. (1997). Effect of frozen storage on the quality of whole fish and fillets of horse mackerel (Trachurus trachurus) and Mediterranean hake (Merluccius mediterraneus). Z Lebensm Unters Forsch A, 204, 405-410

Uddin, M. (2010). Differentiation of fresh and frozen-thawed fish. In: Leo, L. M. L. Nollet, \& F. Toldrá (Eds.), Handbook of seafood and seafood products analysis. CRC Press. (Part V safety, Chapter 37, pp.735-750)

Uddin, M., Okazaki, E., Turza, S., Yumiko, Y., Tanaka, M., \& Fukuda, Y. (2005). Nondestructive 568 visible/NIR spectroscopy for differentiation of fresh and frozen-thawed fish. Journal of Food Science, $70,506-510$

Upton HF. (2015). Seafood fraud. Congressional Research Service, www.crs.gov Document available at: http://nationalaglawcenter.org/wp-content/uploads/assets/crs/RL34124.pdf

Vanhonacker, F., Pieniak, Z., \& Verbeke, W. (2013). European consumer perceptions and barriers for fresh, frozen, preserved and ready-meal fish products. British Food Journal, 115(4), 508-525.

Venugopal, V. (2006). Quick freezing and individually quick frozen products. In: Venugopal (Ed.) Seafood processing. (pp.446) Taylor and Francis, CRC Press, New York. (Chapter 4; pp 95-140)

Zhu, F., Zhang, D., He, Y., Liu, F., \& Sun, D. W. (2013). Application of visible and near infrared hyperspectral imaging to differentiate between fresh and frozen-thawed fish fillets. Food and Bioprocess Technology, 6(10), 2931-2937.

Zhu, S., Ramaswamy, H.S., \& Simpson, B.K. (2004). Effect of high-pressure versus conventional 580 thawing on color, drip loss and texture of Atlantic salmon frozen by different methods. LWT - Food Science and Technology, 37 (3), 291-299 


\section{Highlights}

- A histological procedure to discriminate fresh/frozen-thawed M. merluccius was set up

- Morphological and morphometrical parameters were evaluated on fish muscle tissue

- An operational grid based on four histological parameters was proposed

- The validated procedure is applicable by both specialist analysts and trained operators 
Table 1. Results of the assessment of the morphological parameters on histological slides of fresh (F_MM), conventionally frozen (CF_MM) and Individual Quick Frozen (IQF_MM) M. merluccius specimens. $0=$ muscle organization fully destructured, $1=$ muscle organization partially $(<50 \%)$ destructured, 2 = muscle organization well preserved. V= vacuols; IPM: interstitial preoteinaceus material. * only a vacuole was detected

\begin{tabular}{cccccccccc}
\hline \multirow{2}{*}{$\begin{array}{c}\text { Sample } \\
\text { category }\end{array}$} & \multirow{2}{*}{$\begin{array}{c}\text { Total number } \\
\text { Total \% }\end{array}$} & \multicolumn{2}{c}{$\begin{array}{c}\text { Organization of } \\
\text { muscle structure }\end{array}$} & \multicolumn{2}{c}{ Presence of V } & \multicolumn{2}{c}{ Presence of IPM } \\
\cline { 3 - 11 } & & $\mathbf{0}$ & $\mathbf{1}$ & $\mathbf{2}$ & $\mathbf{0}(\mathbf{A})$ & $\mathbf{1}(\mathbf{P})$ & $\mathbf{0}(\mathbf{A})$ & $\mathbf{1}(\mathbf{P})$ \\
\hline \multirow{2}{*}{ F_MM } & $\mathrm{N}$. & 30 & 0 & 3 & 27 & 27 & $3 *$ & 29 & 1 \\
\cline { 2 - 12 } & $\%$ & 100 & 0 & 10.0 & 90.0 & 90.0 & 10.0 & 97.0 & 3.0 \\
\hline \multirow{2}{*}{ CF_MM } & $\mathrm{N}$ & 30 & 9 & 11 & 10 & 2 & 28 & 1 & 29 \\
\cline { 2 - 11 } & $\%$ & 100 & 30.0 & 36.7 & 33.3 & 6.7 & 93.3 & 3.3 & 96.7 \\
\hline \multirow{2}{*}{ IQF_MM } & $\mathrm{N}$. & 30 & 0 & 0 & 30 & 0 & 30 & 0 & 30 \\
\cline { 2 - 10 } & $\%$ & 100 & 0 & 0 & 100 & 0 & 100 & 0 & 100 \\
\hline
\end{tabular}


Table 2. Contingency table results of Operator $1(\mathrm{Op} 1)$ and Operator $2(\mathrm{Op} 2)$.

\begin{tabular}{cccc|cccc}
\hline \multicolumn{3}{c|}{ Fish category } & \multicolumn{4}{c}{ Fish category } \\
\hline Op1 & Fresh & Frozen & Total & Op 2 & Fresh & Frozen & Total \\
\hline Fresh & 1 & 33 & 34 & Fresh & 0 & 32 & 32 \\
\hline Frozen & 16 & 0 & 16 & Frozen & 17 & 1 & 18 \\
\hline Total & $\mathbf{1 7}$ & $\mathbf{3 3}$ & 50 & Total & $\mathbf{1 7}$ & $\mathbf{3 3}$ & 50 \\
\hline
\end{tabular}


Click here to download high resolution image

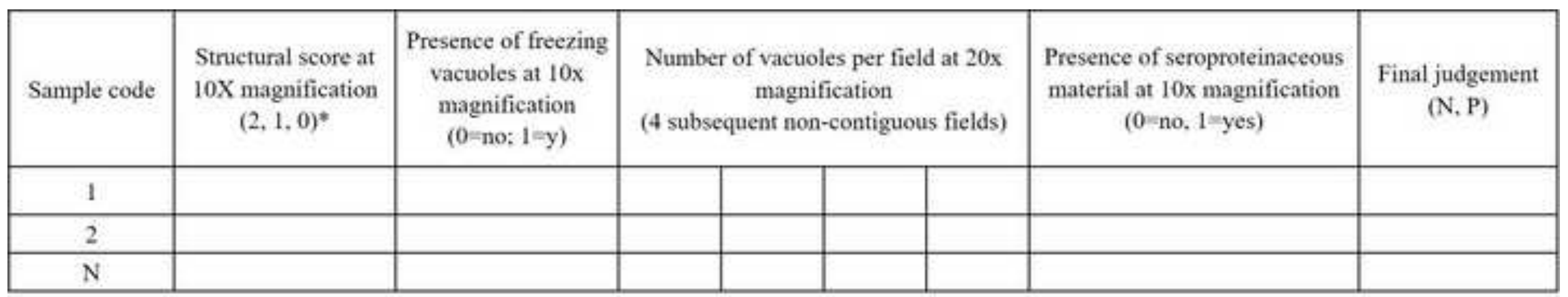


Click here to download high resolution image
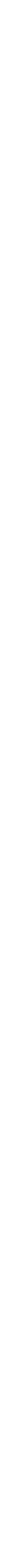
Click here to download high resolution image
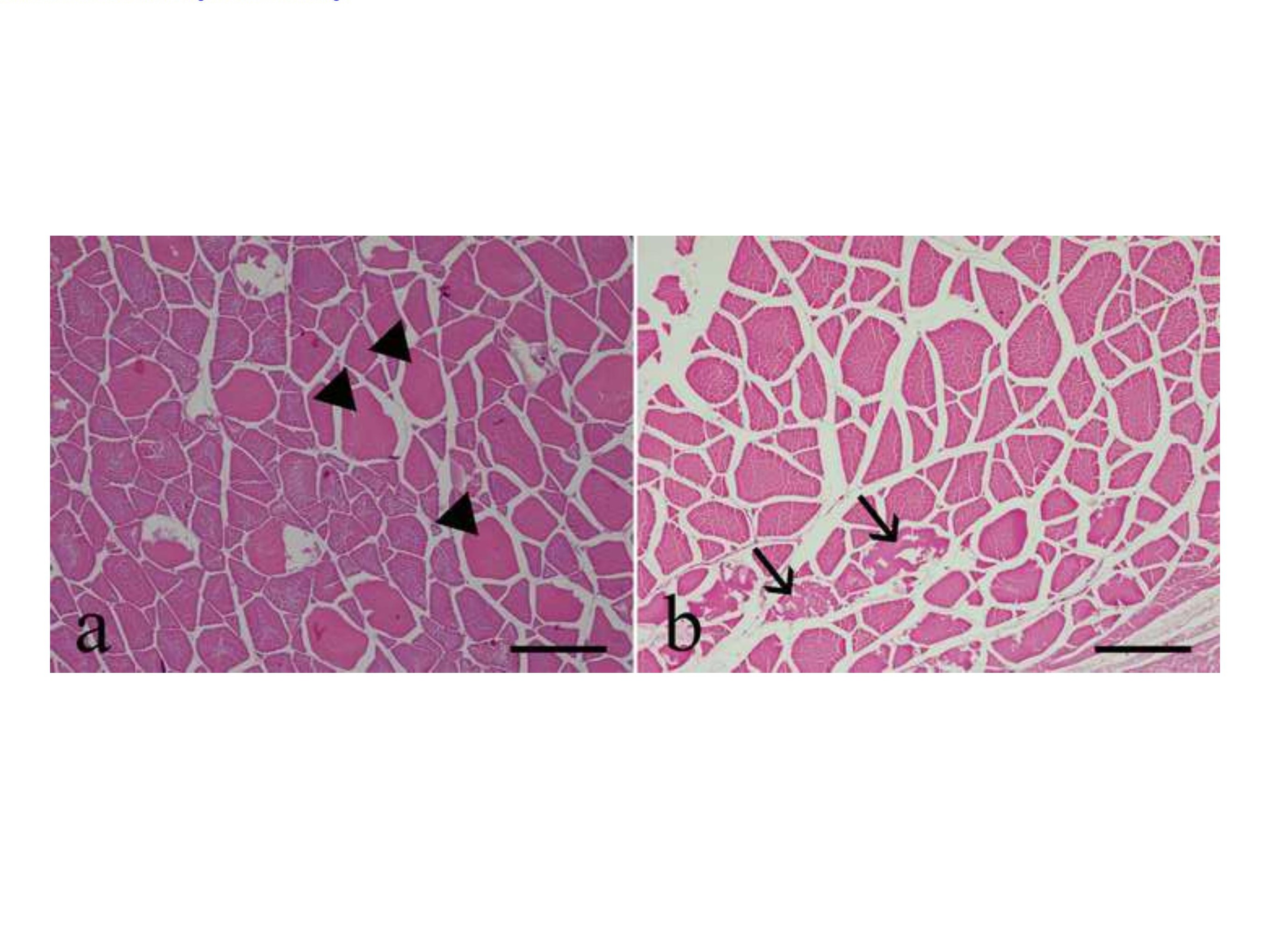
Figure

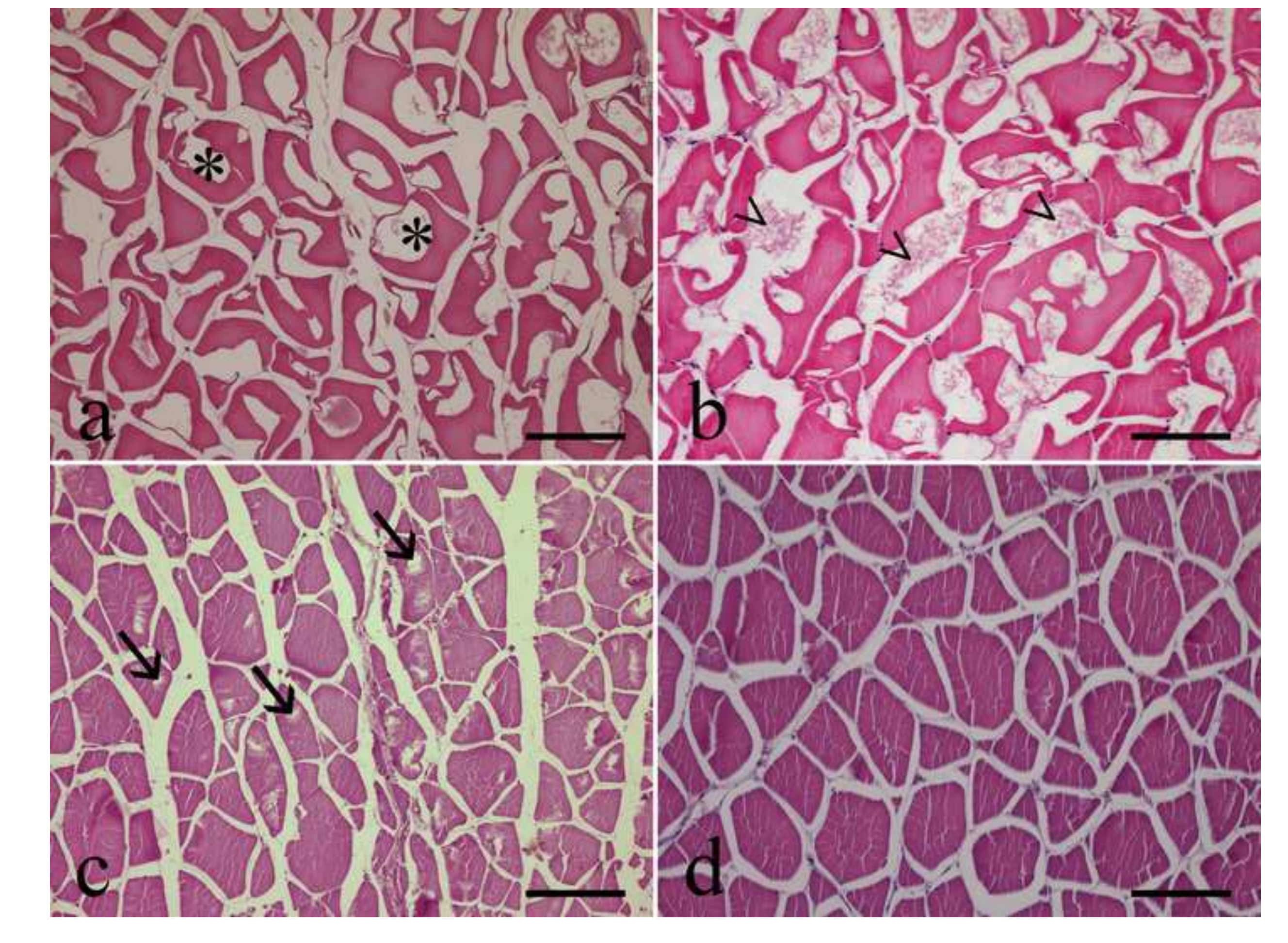

\footnotetext{
Figure
Click here to download high resolution image
}

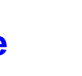


e-component
Click here to download e-component: Table1SM.docx

Click here to download e-component: Table1sM.docx

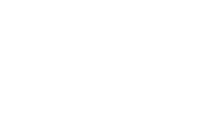

(1)

$\sqrt{2}$

$\sqrt{2}$

$\sqrt{2}$

$\sqrt{2}$

(1)

(1)

(1)

(1) (1) . . . . . . . . . . . . (1)

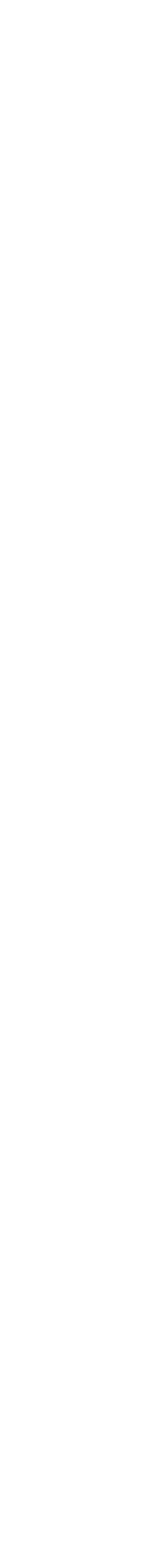
. 